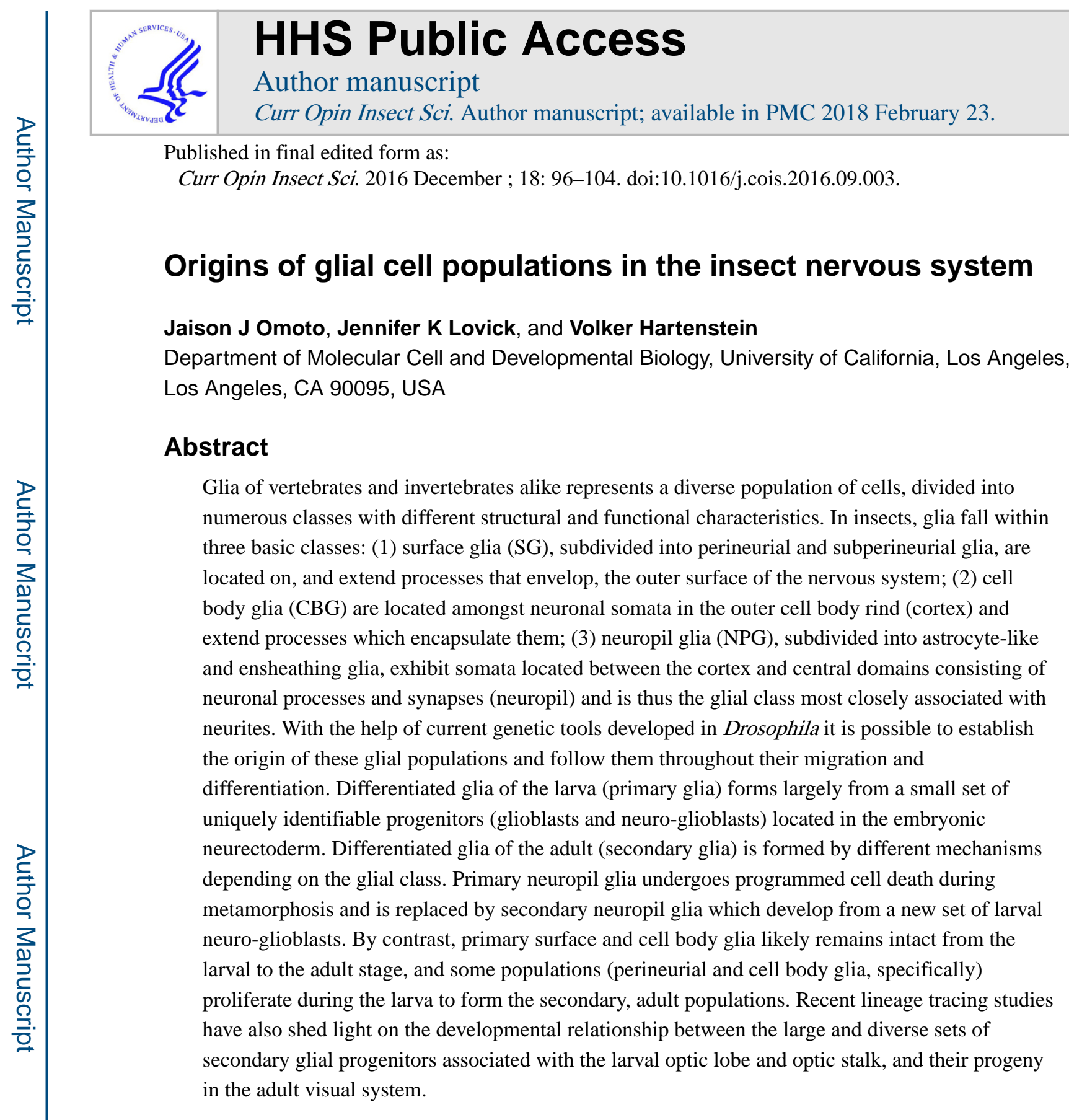

\title{
Neurectodermal origin of glia
}

Cells of the nervous system fall into two main classes of cells: neurons and glia. In vertebrates, glial cells include three major types, astrocytes, oligodendrocytes, and microglia. Astrocytes and oligodendrocytes, together called macroglia, are generated by multipotent neural progenitors that constitute the neuroepithelium lining the neural tube (Bayraktar et al., 2014; Miller, 2002; Fig.1A). During their first rounds of division these cells produce only neurons that segregate from the neuroepithelium (ventricular layer) and form a cellular mantle that matures into nerve tissue (Fig.1B). The neuroepithelial cells left in the ventricular layer become elongated cells, called radial glia. Continued proliferation of 
radial glia located at discrete domains within the neural tube produces oligodendroctye progenitors (OLPs) that spread throughout the CNS and differentiate into oligodendrocytes (Fig.1C). The remainder of radial glia gives rise to astrocytes. Microglial cells are phagocytes combating degenerative processes and infections in the neural tissue. They are derived from blood-forming (hematopoietic) stem cells that migrate into the nervous system during the embryonic period (Lavin et al., 2015; Fig.1A).

In Drosophila, and arthropods in general, glial cells include (1) surface glia (SG) that surrounds the CNS and peripheral nerves and forms the blood-brain barrier; (2) cell body glia (CBG; also called cortex glia), encapsulating neuronal cell bodies which form the outer cellular layer of the CNS; (3) neuropil glia (NPG), located at the interface between the cortex and the neuropil, and forming sheaths around neuropil compartment boundaries, certain long axon bundles, and peripheral nerves (ensheathing glia (EG), wrapping glia), as well as highly branched processes interacting with terminal nerve fibers and synapses [reticular or astrocyte-like glia (ALG)] (Hoyle, 1986; Cantera; 1992; Ito et al., 1995; Pereanu et al., 2005; Awasaki et al., 2008;). Phagocytotic microglial cells are absent; however, blood stem cell-derived macrophages occupy the outer surface of the CNS. Most Drosophila glial cells, along with neurons, are derived from a small number of uniquely identifiable progenitors, called neuroblasts, that delaminate from the neurectoderm of the early embryo (Fig.1G-I). Neuroblasts divide in an asymmetric, stem cell-like pattern, where each division produces a renewed neuroblast, and a ganglion mother cell that becomes postmitotic after one more round of mitosis. Most neuroblasts generate exclusively neurons (which, unlike in vertebrates, outnumber glia by a large margin). Some neuroblasts produce both neurons and glia ("neuro-glioblasts"); very few give rise to glia only ("glioblasts"; Beckervordersandforth et al., 2008; Altenhein et al., 2015). Drosophila exhibits a highly stereotyped nervous system along with a wealth of glial markers and genetic lineage tracing methods allowing for the specific and stable labeling of cells, frequently in a temporallycontrolled manner. As a result, insights into the origin and development of the aforementioned progenitors and their glial progeny, often times at the single-cell level, has been assessed throughout multiple stages of the Drosophila life cycle.

\section{Glia for the larval and adult nervous system: Primary and secondary gliogenesis}

After delamination, embryonic neuroblasts enter an initial phase of proliferation, thus producing the functional neurons of the larval CNS (primary neurons). Many primary neurons, although remodeled during metamorphosis, are retained into adulthood, whereas many others undergo programmed cell death. Along with the neuroblasts, embryonic glioblasts and neuro-glioblasts produce the functional glial cells that differentiate in the late embryo, forming processes around the surface, cell bodies, and neuropil of the larval CNS ("primary glia"; Fig.2A). Glioblasts only generate glia, and express the glial determinants $\mathrm{gcm}$ and repo right after they delaminate from the neurectoderm. Neuro-glioblasts show different modes of gliogenesis (Altenhein et al., 2015; Fig.2B). In type 1 neuro-glioblasts, no early separation of neural vs glial fate takes place; instead, neurons and glial cells derive as siblings from the division of ganglion mother cells. Type 2 neuro-glioblasts switch from 
neurogenesis to gliogenesis at some point in proliferation. Type 3 neuro-glioblasts are defined by the fact that they comprise at least one pure glioblast progenitor. Finally, midline precursors (MPs) constitute a peculiar type of fundamentally-distinct glioblast (mesectodermally-derived) that divide just once to give rise to a subtype of primary neuropil glia, called midline glia (Bossing and Technau, 1994).

A second phase of neurogenesis and gliogenesis takes place during the larval period. Neuroblasts produce secondary neurons that integrate into the circuitry of retained primary neurons during metamorphosis. Thus, a typical neuroblast lineage consists of embryonically-born primary and postembryonically-born secondary neurons. Functional glial populations of the adult ("secondary glia") appear to exhibit a more variable pattern of origin. (1) Secondary neuropil glial cells are generated by a select subpopulation of neuroglioblasts that become active in the larva (Izergina et al., 2009; Omoto et al., 2015; Fig.2A, C, F; see below). (2) By contrast, secondary perineurial and cell body glia are generated by the continued proliferation of their respective primary cell types (Pereanu et al., 2005; Colonques et al., 2007; Awasaki et al., 2008; Kato et al., 2009; Fig.2A, C-E). (3) Primary subperineurial glia do not divide and appear to persist into adulthood.

\section{Neuropil Glia}

The two types of glia in direct contact with nerve processes, astrocyte-like and ensheathing glia, may play different roles in the mature nervous system, but share a common origin. ALGs extend processes into the neuropil and express amino acid transporters, optimally situated to modulate synaptic and/or extrasynaptic processes and thus the circuit properties of neurons, via the uptake of neurotransmitters or other cell-cell signaling pathways (Liu et al., 2014, MacNamee et al., 2016). Ensheathing glia wraps around neuropil compartment boundaries, axon tracts, and fascicles, likely acting as structural insulators of mature neurons (Spindler et al., 2009). Dye injection experiments revealed that a single glioblast, the lateral glioblast (LGB), in each hemisegment gives rise to most of the primary neuropil glia of the embryonic ventral nerve cord, called longitudinal glia based on their association with the longitudinal connectives (Fig.3A-C). Longitudinal glial cells are differentially specified into six ALGs and three EGs, depending on their expression of Notch, Pointed, and Prospero (Peco et al., 2016; Fig.3D-F). Other classes of embryonic primary neuropil glia include repo-negative midline glia (MG), produced from mesectodermally-derived midline precursors (Bossing and Technau, 1994), and nerve root glia (NRG), derived from the neuroglioblast NB1-3 and NB7-4 (Beckervordersandforth et al., 2008; Fig.3A-C). Primary neuropil glia of the brain is thought to be derived from the $\mathrm{Td} 7$ neuroblast (nomenclature according to Urbach and Technau, 2003), based on its expression of glial specific genes, and its close proximity to the longitudinal glia equivalent of the brain, the basal procephalic longitudinal glia (BPLG; Hartenstein et al., 1998; Omoto et al., 2015; Fig.3G).

The number of differentiated primary neuropil glia remains constant throughout larval development. These cells increase in size presumably to account for the growing neuropil volume (Omoto et al., 2015). Temporally-controlled lineage tracing demonstrated that primary astrocyte-like glia, and likely ensheathing glia as well, undergoes programmed cell death during metamorphosis and is not retained into adulthood (Fig.3H-J). Although the 
number of differentiated primary neuropil glial cells does not change throughout the larval period, clone induction via MARCM (mosaic analysis with a repressible cell marker) during the secondary phase of neuroblast proliferation revealed that repo-positive neuropil glial precursors appear in large numbers as a result of the proliferation of a few secondary neuroglioblasts (Izergina et al., 2009; Viktorin et al., 2011; Omoto et al. 2015; Fig.2C, F). Lineage tracing experiments revealed that these secondary neuropil glial precursors migrate from their site of origin in the brain cortex towards the neuropil, proliferate, spread out tangentially around the neuropil surface/between neuropil compartments, and differentiate into secondary neuropil glia. The analogous process occurs in select, yet to be identified neuro-glioblasts of the ventral nerve cord. Although primary and secondary neuropil glia exhibit fundamental similarities, they differ in size and in gene expression profiles (Omoto et al., 2015; Huang, et al., 2015). Primary midline glia of the ventral nerve cord show an unusual behavior: like other neuropil glia they degenerate during metamorphosis, but they proliferate profusely prior to this event, possibly to assist in circuit formation (Awad and Truman, 1997).

\section{Surface Glia}

Surface glia surrounding the larval and adult brain, as well as all peripheral nerves, can be subdivided into perineurial (PNG) and subperineurial glia (SPG). Subperineurial cells form conspicuous septate junctions (the invertebrate equivalent of tight junctions) and are thereby primarily important to generate a blood-brain barrier that prevents unregulated passage of molecules between the nervous system and hemolymph (Limmer et al., 2014). Perineurial glial is also involved in the blood-brain barrier, even though the mechanism by which it participates in this function is not clear, since perineurial glial cells do not form a complete sheath connected by septate junctions around the brain. Furthermore, in the early larva, PNGs are lacking at most locations; they only form a relatively dense cover after a phase of intense proliferation in the late larva (Pereanu et al., 2005; Avet-Rochex et al., 2014; Fig. 2D). Recent evidence also suggests a role of surface glia in regulation of neuronal metabolism, as well as the coupling of systemic metabolism to brain development (Chell and Brand, 2010, Volkenhoff et al., 2015).

Primary surface glia of the ventral nerve cord is derived from five neuro-glioblasts (Type 1: NB1-1, NB2-2, NB5-6; Type 2: NB1-3, NB7-4), located at the boundary between two adjacent segments (Beckervordersandforth et al., 2008; Altenhein et al., 2015; Fig.3A). The contribution of primary surface glia to the insulating layers around the peripheral nerves have been revealed by Flybow, a stable, multicolor labeling technique (von Hilchen et al., 2008, 2013), and its origins traced back to NB2-5 and NB5-6, or NB1-3, respectively (Fig. $3 \mathrm{~A}, \mathrm{C})$. In addition, a subset peripheral nerve surface glia is produced by sensory organ progenitor cells which form outside the neurectoderm (Fig.3C). How surface glia is specified into the perineurial and subperineurial type is unknown. During the larval period, primary subperineurial cells increase in size, but not number, due to polyploidization, akin to what has been observed for primary neuropil glia (Unhavaithaya and Orr-Weaver, 2012). We posit that these cells are retained throughout metamorphosis and become the functional subperineurial glia of the adult brain. This hypothesis is supported by the developmental fate of subperineurial glia of the optic stalk (called carpet glia), which is also retained in the adult 
optic lobe (Edwards et al., 2012; Fig.3K). Perineurial glia, in contrast to subperineurial glia, continually proliferates throughout the larval period, and forms the perineurial glia of the adult brain (Awasaki et al., 2008). Proliferating perineurial cells may also contribute to other glial types, as observed for the the perineurial glia of the optic stalk, which differentiates first into a cell type that envelops the retinal axons invading the optic lobe ("wrapping glia"; Bauke et al., 2015), and ultimately gives rise to a type of cortex glia of the optic lobe (distal satellite glia; Edwards et al., 2012; Fig.3K).

\section{Cell body glia}

Cell body glia ensheathes individual neuronal somata, and in doing so, structurally stabilize them while simultaneously regulating whole nervous system excitability (Melom and Littleton, 2013). Primary cell body glial cells of the ventral nerve cord, numbering 3 per hemisegment, are generated by the neuro-glioblasts NB6-4 (Type 3) and 7-4 (Type 2; Fig. 3A-C; Altenhain et al., 2015). For the brain, the specific neuro-glioblasts which give rise to the primary cell body glia, as well as surface glia, have not yet been identified. However, clusters of repo-positive cells that include precursors of both cell types were mapped to a domain at the border between protocerebrum and deuterocerebrum, and to the dorsal edge of the protocerebrum, respectively (Hartenstein et al., 1998; Fig.3G). Like perineurial glia, cell body glia enters a phase of proliferation during the late larval period. MARCM analysis revealed that proliferation for both glial types depends on several signaling pathways, among them Hippo (Reddy and Irvine, 2011), InR/TOR, and FGF (Avet-Rochex et al., 2012A, 2014). The ligands activating these pathways appear to act locally. Thus, the FGF cognate Pyramus (Pyr), is secreted by neighboring glial cells (in case of perineurial glia) or neurons (in case of cell body glia). Likewise, the signal activating the InR/TOR pathway, Drosophila insulin-like peptide 6 (Dilp-6), is found in glial cells (Avet-Rochex et al., 2012).

\section{Glia of the optic lobe}

The optic lobe, which receives retinotopically ordered input from the compound eye, develops postembryonically from two neuroepithelial optic anlagen, called outer and inner optic anlagen, which, initially, grow by symmetric division, followed by their conversion into a large number of asymmetrically dividing neuroblasts (Fischbach and Hiesinger, 2008; Ngo et al., 2010). Glia of the optic lobe exhibits numerous morphologically distinct subclasses that are not evident in the central brain or ventral nerve cord. For example, cortex glia of the lamina include distal and proximal satellite glia; neuropil glia has epithelial glia and marginal glia, as well as outer chiasm glia (Edwards et al., 2012). Surface glia of the optic lobe is derived from the proliferating perineurial glia surrounding the larval brain and optic stalk (Fig.3K). Neuropil and cell body glia of the lamina are formed by four distinct populations of glial progenitors that derive from the glial precursor zone (Chotard and Salecker, 2005; Edwards et al., 2012), a subdomain of the outer optic anlage (Fig.3K). Perineurial glia of the optic stalk, which first differentiates into wrapping glia (Bauke et al., 2015), also contributes to the lamina cortex glia (distal satellite glia; Edwards et al., 2012). Precursors of neuropil and cortex glia of the other optic ganglia (medulla, lobula, lobula plate) are born at a later stage during the asymmetric division of neuro-glioblasts of the outer and inner optic anlage (Hartenstein, 2011; Edwards et al., 2012; Fig.3K). 


\section{Hemocytes and the nervous system}

Blood cells populating the body cavity of the Drosophila larva (primary or "primitive" hemocytes) are formed in the head mesoderm of the early embryo (Tepass et al., 1994). $\mathrm{Gcm}$, the transcription factor specifying glial fate in the progeny of neuro-glioblasts, is also expressed and required for mesodermal cells to adopt a hemocyte fate (Bernardoni et al., 1997; Lebestky et al., 2000; Cattenoz et al., 2016). From their anterior location, hemocytes migrate along the surface of the nervous system and other inner organs, laying down components of the extracellular matrix (Fig.4A-D).

In addition, a major role of hemocytes (similar to vertebrate microglia) lies in phagocytosing apoptotic cell bodies resulting from programmed cell death. Loss or altered function of hemocytes results in severe defects in nervous system development (Sears et al., 2003; Oloffson and Page, 2005). The removal of cellular debris in the CNS, which in vertebrates is carried out by microglia, is a function shared between hemocytes and (macro-)glia in Drosophila. In the fly embryo, primary neuropil glia and surface glia, in addition to hemocytes, act as macrophages (Sonnenfeld and Jacobs, 1995). Postembryonically, neuropil glia exhibits functions reminiscent of vertebrate microglia, depending on the context; astrocyte-like cells clear neuronal debris as a consequence of neuronal remodeling during metamorphosis, whereas ensheathing glia clears neuronal debris during neurite injury in the adult brain (Doherty et al., 2009, Tasdemir-Yilmaz and Freeman, 2014). Interestingly, by triggering an immune response (activation of the Imd pathway) in glial cells resulted in the appearance of a non-neural/non-glial cell type type within the brain neuropil that appeared to be migratory, and expressed markers for phagocytosis (Stratoulias and Heino, 2015). It is tempting to draw a close functional and ontogenetic connection between these cells, derived from immune-challenged glia or hemocytes (or both), and vertebrate microglia.

The conservation of many molecular pathways controlling their development and functions suggest that hemocytes in arthropods and blood cells in vertebrates, including microglia and other tissue-resident-macrophages, are homologous cell types. Certain types of macroglia, such as vertebrate astrocytes and Drosophila astrocyte-like cells, also share many functional properties, but it is currently uncertain whether this is based on homology on the cellular level. Thus, glial cells are absent from the nervous system of many basal metazoans (Hartline, 2011), and it is doubtful that the common bilaterian ancestor possessed glial cells. Nevertheless, recent analyses provide an increasing body of evidence that molecular pathways controlling functions such as phagocytosis, transmitter re-uptake, or response to injury are shared between glial cells of vertebrates and Drosophila (reviewed in Freeman, 2015), which would argue for a deep homology, where pre-existing, homologous gene networks controlling such functions were recruited into cells that then became glial cells. In all, due to its genetic amenability, progress to understand the development and function of Drosophila glia has accelerated and will surely continue to yield valuable insight into glial biology in general.

\section{References}

*. Altenhein B. Glial cell progenitors in the Drosophila embryo. Glia. 2015; 63:1291-1302. This review discusses the origins of glial cell progenitors with an emphasis on progenitors of glial 
subtypes in the CNS and PNS, but not midline glia. DOI: 10.1002/glia.22820 [PubMed: 25779863]

*. Altenhein B, Cattenoz PB, Giangrande A. The early life of a glial cell. Wiley Interdiscip Rev Dev Biol. 2016; 5:67-84. This review discusses the origin of glial progenitors in the embryo as well as embryonic and postembryonic glial subtypes including perineurial, subperineurial, cortex, neuropil, and lateral glia. DOI: 10.1002/wdev.200 [PubMed: 26224590]

Awad TA, Truman JW. Postembryonic development of the midline glia in the CAN of Drosophila: proliferation, programmed cell death, and endocrine regulation. Dev Biol. 1997; 187:283-297. DOI: 10.1006/dbio.1997.8587 [PubMed: 9242424]

Awasaki T, Lai SL, Ito K. Lee T: Organization and postembyonic development of glial cells in the adult central brain of Drosophila. J Neurosci. 2008; 28:13742-13753. DOI: 10.1523/JNEUROSCI. 4844-08.2008 [PubMed: 19091965]

Avet-Rochex A, Kaul AK, Gatt AP, McNeill H, Bateman JM. Concerted control of gliogenesis by InR/TOR and FGF signalling in the Drosophila post-embryonic brain. Development. 2012; 139:2763-2772. DOI: 10.1242/dev.074719 [PubMed: 22745312]

*. Avet-Rochex A, Maierbrugger KT, Bateman JM. Glial enriched gene expression profiling identifies novel factors regulating the proliferation of specific glial subtypes in the Drosophila brain. Gene Expr Patterns. 2014; 16:61-68. Gene expression profiling identified subtype-specific genes in perineurial glia and cortex glia: tramtrack69 positively regulates proliferation in both subtypes and foxO inhibits proliferation in perineurial glia. DOI: 10.1016/j.gep.2014.09.001 [PubMed: 25217886]

*. Bauke AC, Sasse S, Matzat T, Klämbt C. A transcriptional network controlling glial development in the Drosophila visual system. Development. 2015; 142:2184-2193. In the developing eye, the DNA-binding protein Dref positively regulates proliferation of perineurial glia via the transcription factor Pdm3. Both Dref and the FGF signaling pathway control differentiation of perineurial glia into wrapping glia via the homeodomain protein Cut. DOI: 10.1242/dev.119750 [PubMed: 26015542]

Bayraktar OA, Fuentealba LC, Alvarez-Buylla A, Rowitch DH. Astrocyte development and heterogeneity. Cold Spring Harb Perspect Biol. 2014; 7:a020362.doi: 10.1101/cshperspect.a020362 [PubMed: 25414368]

Beckervordersandforth RM, Rickert C, Altenein B, Technau GM. Subtypes of glial cells in the Drosophila embryonic ventral nerve cord as related to lineage and gene expression. Mech Dev. 2008; 125:542-557. DOI: 10.1016/j.mod.2007.12.004 [PubMed: 18296030]

Bernardoni R, Vivancos V, Giangrande A. glide/gcm is expressed and required in the scavenger cell lineage. Dev Biol. 1997; 191:118-130. DOI: 10.1006/dbio.1997.8702 [PubMed: 9356176]

Bodmer R, Carretto R, Jan YN. Neurogenesis of the peripheral nervous system in Drosophila embryos: DNA replication patterns and cell lineages. Neuron. 1989; 3(89):21-32. 90112-8. DOI: 10.1016/0896-6273 [PubMed: 2515889]

Bossing T, Technau GM. The fate of the CNS midline progenitors in Drosophila as revealed by a new method for single cell labeling. Development. 1994; 120:1895-1906. [PubMed: 7924995]

Cantera R. Glial cells in adult and developing prothoracic ganglion of the hawk moth Manduca sexta. Cell Tissue Res. 1993; 272:93-108.

*. Cattenoz PB, Giangrande A. New insights in the clockwork mechanisms regulating lineage specification: Lessons from the Drosophila nervous sytem. Dev Dyn. 2015; 244:332-341. Review of complex regulation of the glial determinant $\mathrm{Gcm}$ with specific emphasis on transcriptional regulation, epigenetic regulation, post-transcriptional modifications, and $\mathrm{Gcm}$ autoregulation. DOI: 10.1002/dvdy.24228 [PubMed: 25399853]

*. Cattenoz PB, Popkova A, Southall TD, Aiello G, Brand AH, Giangrande A. Functional conservation of the Glide/Gcm regulatory network controlling glia, hemocyte, and tendon cell differentiation in Drosophila. Genetics. 2016; 202:191-219. Performed DamID genome-wide screen to identify transcriptional targets of Glide/Gcm; found components of the Notch, Hedgehog, and JAK/STAT signaling pathways as well as genes important for cell adhesion and axonal navigation. Also, demonstrate that many of the Glide/Gcm targets are common in flies and vertebrates. DOI: 10.1534/genetics.115.182154 [PubMed: 26567182] 
Chell JM, Brand AH. Nutrition-responsive glia control exit of neural stem cells from quiescence. Cell. 2010; 143:1161-1173. DOI: 10.1016/j.cell.2010.12.007 [PubMed: 21183078]

Chotard C, Salecker I. Glial cell development and function in the Drosophila visual system. Neuron Glia Biol. 2007; 3:17-25. DOI: 10.1017/S1740925X07000592 [PubMed: 18333286]

Colonques J, Ceron J, Tejedor FJ. Segregation of postembryonic neuronal and glial lineages inferred from a mosaic analysis of the Drosophila larval brain. Mech Dev. 2007; 124:327-340. DOI: 10.1016/j.mod.2007.01.004 [PubMed: 17344035]

Dearborn R Jr, Kunes S. An axon scaffold induced by retinal axons directs glia to destinations in the Drosophila optic lobe. Development. 2004; 131:2291-2303. DOI: 10.1242/dev.01111 [PubMed: 15102705]

DeSalvo MK, Hindle SJ, Rusan ZM, Orng S, Eddison M, Halliwill K, Bainton RJ. The *Drosophila surface glia transcriptome: evolutionary conserved blood-brain barrier processes. Front Neurosci. 2014; 8:346. Microarray of surface glia revealed enrichment for ATP-binding cassette and solute carrier transporters, cell adhesion molecules, signaling molecules, and genes important for metabolism. Many of these genes are conserved and also enriched in blood-brain barrier cells in mouse. doi: 10.3389/fnins.2014.00346 [PubMed: 25426014]

Doherty J, Logan MA, Tasdemir OE, Freeman MR. Ensheathing glia function as phagocytes in the adult Drosophila brain. J Neurosci. 2009; 29:4768-4781. DOI: 10.1523/JNEUROSCI. 5951-08.2009 [PubMed: 19369546]

Edwards TN, Meinertzhagen IA. The functional organisation of glia in the adult brain of Drosophila and other insects. Prog Neurobiol. 2010; 90:471-497. DOI: 10.1016/j.pneurobio.2010.01.001 [PubMed: 20109517]

Edwards TN, Nuschke AC, Nern A, Meinertzhagen IA. Organization and metamorphosis of glia in the Drosophila visual system. J Comp Neurol. 2012; 520:2067-2085. DOI: 10.1002/cne.23071 [PubMed: 22351615]

Fischbach KF, Hiesinger PR. Optic lobe development. Adv Exp Med Biol. 2008; 628:115-136. DOI: 10.1007/978-0-387-78261-4_8 [PubMed: 18683642]

Hakim Y, Yaniv SP, Schuldiner O. Astrocytes play a key role in Drosophila mushroom body axon pruning. PLoS One. 2014; 9:e86178.doi: 10.1371/journal.pone.0086178 [PubMed: 24465945]

Hartenstein V. Morphological diversity and development of glia in Drosophila. Glia. 2011; 59:12371252. DOI: 10.1001/glia.21162 [PubMed: 21438012]

Hartenstein V, Nassif C, Lekven A. Embryonic development of the Drosophila brain. II. Pattern of glial cells. J Comp Neurol. 1998; 402:32-47. [PubMed: 9831044]

Hartline DK. The evolutionary origins of glia. Glia. 2011; 59:1215-1236. DOI: 10.1002/glia.21149 [PubMed: 21584869]

Hoyle G, Williams M, Phillips C. Functional morphology of insect neuronal cell-surface/glial contacts: the trophospongium. J Comp Neurol. 1986; 246:113-128. DOI: 10.1002/cnd.902460108 [PubMed: 3700714]

*. Huang Y, Ng FS, Jackson FR. Comparison of larval and adult Drososphila astrocytes reveals stagespecific gene expression profiles. G3 (Bethesda). 2015; 5:551-558. Genome-wide expression profiling of larval and adult astrocyte-like glia identified genes potentially important for mediating functions in adult astrocyte-like glia. Both were enriched for genes important for metabolism and energy production, but adult specific cells were also enriched for genes involved in chromatin modification and vesicle-mediated trafficking or secretion. DOI: 10.1534/ g3.114.016162 [PubMed: 25653313]

Izergina N, Balmer J, Bello B, Reichert H. Postembryonic development of transit amplifying neuroblast lineages in the Drosophila brain. Neural Dev. 2009; 4:44.doi: 10.1186/1749-8104-4-44 [PubMed: 20003348]

Jones BW. Transcriptional control of glial cell development in Drosophila. Dev Biol. 2005; 278:265273. DOI: 10.1016/j.ydbio.2004.11.022 [PubMed: 15680348]

Kato K, Awasaki T, Ito K. Neuronal programmed cell death induces glial cell division in the adult Drosophila brain. Development. 2009; 136:51-59. DOI: 10.124/dev.023366 [PubMed: 19019992]

*. Kim HJ, Ahn HJ, Lee S, Kim JH, Jark J, Jeon SH, Kim SH. Intrinsic dorsoventral patterning and extrinsic EGFR signaling genes control glial cell development in the Drosophila nervous system. 
Neuroscience. 2015; 307:242-252. Lateral glial cells derived from lateral neuroectoderm in the embryo are specified by the dorsoventral patterning genes vnd, ind, and msh as well as the EGFR signaling pathway. The dorsoventral patterning genes achieve this through regulation of the gliaspecific genes gcm and repo. DOI: 10.1016/j:neuroscience.2015.08.049 [PubMed: 26318336]

*. Kis V, Barti B, Lippai M, Sass M. Specialized cortex glial cells accumulate lipid droplets in Drosophila melanogaster. PLoS One. 2015; 10:e0131250. Lipid droplets are found in glia of the larval nervous system, but are enriched in cortex glial cells near the brain surface. Lipid droplets are in greatest abundance at the onset of metamorphosis. doi: 10.1371/journal.pone. 0131250 [PubMed: 26148013]

Klaes A, Menne T, Stollewerk A, Scholz H, Klämbt C. The Ets transcription factor encoded by the Drosophila gene pointed direct glial cell differentiation in the embryonic CNS. Cell. 1994; 78:149-160. DOI: 10.1016/0092-8674(94)90581-9 [PubMed: 8033206]

Klämbt C, Goodman CS. Role of the midline glia and neurons in the formation of the axon commissures in the central nervous system of the Drosophila embryo. Ann N Y Acad Sci. 1991; 633:142-159. DOI: 10.1111/j.1749-6632.1991.tb15604.x [PubMed: 1789544]

Lavin Y, Mortha A, Rahman A, Merad M. Regulation of macrophage development and function in the peripheral tissues. Nat Rev Immunol. 2015; 15:731-744. DOI: 10.1038/nri3920 [PubMed: 26603899]

Lebestky T, Chang T, Hartenstein V, Banerjee U. Specification of Drosophila hematopoietic lineage by conserved transcription factors. Science. 2000; 288:146-149. DOI: 10.1126/science.288.5463.146 [PubMed: 10753120]

*. Li HH, Kroll JR, Lennox SM, Ogundeyi O, Jeter J, Depasquale G, Truman JW. A GAL4 driver resource for developmental and behavioral studies on the larval CNS of Drosophila. Cell Rep. 2014; 8:897-906. Describe larval CNS expression patters of GAL4 lines based on cis-regulatory regions. Many of these lines are expressed in glia, including astrocyte-like glia, which enables identification that these cells derive from glia which surrounds the developing leg neuropil in the ventral CNS. DOI: 10.1016/j.celrep.2014.06.065 [PubMed: 25088417]

Limmer S, Weiler A, Volkenhoff A, Babatz F, Klämbt C. The Drosophila blood-brain barrier: development and function of a glial endothelium. Front Neurosci. 2014; 8:365.doi: 10.3389/fnins. 2014.00365 [PubMed: 25452710]

Liu H, Zhou B, Yan W, Lei Z, Zhao X, Zhang K, Guo A. Astrocyte-like glial cells physiologically regulate olfactory processing through the modification of ORN-PN synaptic strength in Drosophila. Eur J Neurosci. 2014; 40:2744-2754. DOI: 10.1111/ejn.12646 [PubMed: 24964821]

Macnamee SE, Liu KE, Gerhard S, Tran CT, Fetter RD, Cardona A, Tolbert LP, Oland LA. Astrocytic glutamate transport regulates a Drosophila CNS synapse that lacks astrocyte ensheathment. J Comp Neurol. 2016; 524:1979-1998. DOI: 10.1002/cne.24016 [PubMed: 27073064]

Matzat T, Siegliatz F, Kottmeier R, Babatz F, Engelen D, Klämbt C. Axonal wrapping in the Drosophila PNS is controlled by glia-derived neuregulin homolog Vein. Development. 2015; 142:1336-1345. DOI: 10.1242/dev.116616 [PubMed: 25758464]

Meinertzhagen, IA., Hanson, TE. The development of the optic lobe. In: Martinez Arias, A., Bate, M., editors. The development of Drosophila melanogaster. New York: Cold Spring Harbor Press; 1993. p. 1363-1491.

Melom JE, Littleton JT. Mutation of a NCKX eliminates glial microdomain calcium oscillations and enhances seizure susceptibility. J Neurosci. 2013; 33:1169-1178. [PubMed: 23325253]

Meyer S, Schmidt I, Klämbt C. Glia ECM interactions are required to shape the Drosophila nervous system. Mech Dev. 2014; 133:105-116. DOI: 10.1016/j.mod.2014.05.003 [PubMed: 24859129]

Miller RH. Regulation of oligodendrocyte development in the vertebrate CNS. Prog Neurobiol. 2002; 67:451-467. DOI: 10.1016/S0301-0082(02)00058-8 [PubMed: 12385864]

Ngo KT, Wang J, Junker M, Kriz S, Vo G, Asem B, Olson JM, Banerjee U, Hartenstein V. Concomitant requirement for Notch and Jak/Stat signaling during neuro-epithelial differentiation in the Drosophila optic lobe. Dev Biol. 2010; 346:284-295. DOI: 10.1016/j.ydbio.2010.07.036 [PubMed: 20692248] 
Olofsson B, Page DT. Condensation of the central nervous system in embryonic Drosophila is inhibited by blocking hemocyte migration or neural activity. Dev Biol. 2005; 279:233-243. DOI: 10.1016/j.ydbio.2004.12.020 [PubMed: 15708571]

**. Omoto JJ, Yogi P, Hartenstein V. Origin and development of neuropil glia of the Drosophila larval and adult brain: Two distinct glial populations derived from separate progenitors. Dev Biol. 2015; 404:2-20. Neuropil glia (astrocyte-like and ensheathing) of the larval brain derive from progenitors located in the basal part of the embryonic brain whereas adult-specific astrocyte-like and ensheathing glia arise from Type II neuroglioblasts beginning at the late larval stage. DOI: 10.1016/j.ydbio.2015.03.004 [PubMed: 25779704]

**. Peco E, Davla S, Camp DM, Stacey S, Landgraf M, van Meyel DJ. Drosophila astrocytes cover specific territories of the CNS neuropil and are instructed to differentiate by Prospero, a key effector of Notch. Development. 2016; 143:1170-1181. Larval astrocytes, ensheathing glia, and ensheathing/wrapping glia derive from embryonic longitudinal glia. Astrocytes are specified through a combination of Notch signaling and its effector gene Prospero as well as the Ets transcription factor Pointed P1. DOI: 10.1242/dev.133165 [PubMed: 26893340]

Pereanu W, Shy D, Hartenstein V. Morphogenesis and proliferation of the larval brain glia in Drosophila. Dev Biol. 2005; 283:191-203. DOI: 10.1016/j.ydbio.2005.04.024 [PubMed: 15907832]

Reddy BV, Irvine KD. Regulation of Drosophila glial cell proliferation by Merlin-Hippo signaling. Development. 2011; 138:5201-5212. DOI: 10.1242/dev.069385 [PubMed: 22069188]

Sears HC, Kennedy CJ, Garrity PA. Macrophage-mediated corpse engulfment is required for normal Drosophila CNS morphogenesis. Development. 2003; 130:3557-3565. DOI: 10.1242/dev.00586 [PubMed: 12810602]

Sonnenfeld MJ, Jacobs JR. Macrophages and glia participate in the removal of apoptotic neurons from the Drosophila embryonic nervous system. J Comp Neurol. 1995; 359:644-652. DOI: 10.1002/ cne.903590410 [PubMed: 7499553]

Spindler SR, Ortiz I, Fung S, Takashima S, Hartenstein V. Drosophila cortex and neuropile glia influence secondary axon tract growth, pathfinding, and fasciculation in the developing larval brain. Dev Biol. 2009; 334:355-368. DOI: 10.1016/j.ydbio.2009.07.035 [PubMed: 19646433]

Stacey SM, Muraro NI, Peco E, Labbé A, Thomas GB, Baines RA, van Meyel DJ. Drosophila glial glutamate transporter Eaat 1 is regulated by fringe-mediated notch signaling and is essential for larval locomotion. J Neurosci. 2010; 30:14446-14457. DOI: 10.1523/JNEUROSCI.1021-10.2010 [PubMed: 20980602]

Stork T, Engelen D, Krudewig A, Silies M, Bainton RJ, Klämbt C. Organization and function of the blood-brain barrier in Drosophila. J Neurosci. 2008; 28:587-597. DOI: 10.1523/JNEUROSCI. 4367-07.2008 [PubMed: 18199760]

*. Stork T, Sheehan A, Tasdemir-Yilmaz OE, Freeman MR. Neuron-glia interactions through the Heartless FGF receptor signaling pathway mediate morphogenesis of Drosophila astrocytes. Neuron. 2014; 83:388-403. FGF signaling in the neuropil directs the morphogenesis of astrocytes. The FGF signaling pathway is activated in astrocytes by the FGF ligands Pyramus and Thisbe, which are expressed by neighboring neurons. DOI: 10.1016/j.neuron.2014.06.026 [PubMed: 25033182]

Stratoulias V, Heino TI. MANF silencing, immunity induction or autophagy trigger an unusual cell type in metamorphosing Drosophila brain. Cell Mol Life Sci. 2015; 72:1989-2004. Identified a novel cell type in the brain that expresses markers common to macrophages/hemocytes in Drosophila and microglia in vertebrates and is migratory; this cell type only appears after induction of the immune response, autophagy or silencing of the neurotrophic factor DmMANF in all central brain glial cells. DOI: 10.1007/s00018-014-1789-7 [PubMed: 25511196]

Tepass U, Fessler LI, Aziz A, Hartenstein V. Embryonic origin of hemocytes and their relationship to cell death in Drosophila. Development. 1994; 120:1829-1837. [PubMed: 7924990]

Thomas GB, van Meyel DJ. They glycosyltransferase Fringe promotes Delta-Notch signaling between neurons and glia, and is required for subtype-specific glial gene expression. Development. 2007; 134:591-600. DOI: 10.1242/dev.02754 [PubMed: 17215308] 
Udolph G, Rath P, Chia W. A requirement for Notch in the genesis of a subset of glial cells in the Drosophila embryonic central nervous system which arise through asymmetric divisions. Development. 2001; 128:1457-1466. [PubMed: 11262244]

Unhavaithaya Y, Orr-Weaver TL. Polyploidization of glia in neural development links tissue growth to blood-brain barrier integrity. Genes Dev. 2012; 26:31-36. DOI: 10.1101/gad.177436.111 [PubMed: 22215808]

Viktorin G, Riebli N, Popkova A, Giangrande A, Reichert H. Multipotent neural stem cells generate glial cells of the central complex through transit amplifying intermediate progenitors in Drosophila brain development. Dev Biol. 2011; 356:553-565. DOI: 10.1016/j.ydbio.2011.06.013 [PubMed: 21708145]

Von Hilchen CM, Beckervordersandforth RM, Rickert C, Technau GM, Altenhein B. Identity, origin, and migration of peripheral glial cells in the Drosophila embryo. Mech Dev. 2008; 125:337-352. DOI: 10.1016/j.mod.2007.10.010 [PubMed: 18077143]

Von Hilchen CM, Bustos AE, Giangrande A, Technau GM, Altenhein B. Predetermined embryonic glial cells form the distinct glial sheaths of the Drosophila peripheral nervous system. Development. 2013; 140:3657-3668. DOI: 10.1242/dev.093245 [PubMed: 23903191]

Watson JD, Wheeler SR, Stagg SB, Crews ST. Drosophila hedgehog signaling and engrailed-runt mutual repression direct midline glia to alternative ensheathing and non-ensheathing fates. Development. 2011; 138:1285-1295. DOI: 10.1242/dev.056895 [PubMed: 21350018]

Hoyle G. Glial cells of an insect ganglion. J Comp Neurol. 1986; 246:85-103. DOI: 10.1002/cne. 902460106 [PubMed: 3700719]

Ito K, Urban J, Technau GM. Distribution, classification, and development of Drosophila glial cells in the late embryonic and early larval ventral nerve cord. Roux's archives of developmental biology. 1995; 204:284-307. DOI: 10.1007/BF02179499

Urbach R, Technau GM. Molecular markers for identified neuroblasts in the developing brain of Drosophila. Development. 2003; 130:3621-3637. DOI: 10.1242/dev.00533 [PubMed: 12835380]

*. Volkenhoff A, Weiler A, Letzel M, Stehling M, Klämbt C, Schirmeier S. Glial glycolysis is essential for neuronal survival in Drosophila. Cell Metab. 2015; 22:437-447. Perineurial glia of the bloodbrain barrier metabolize sugars to form secreted alanine and lactate. Without glycolysis in the perineurial glia, neurons die, suggesting that sugars provided by perineurial glia are important for neuronal survival. DOI: 10.1016/j.cmet.2015.07.006 [PubMed: 26235423]

*. Tasdemir-Yilmaz OE, Freeman MR. Astrocytes engage unique molecular programs to engulf pruned neuronal debris from distinct subsets of neurons. Genes Dev. 2014; 28:20-33. During metamorphosis, astrocytes become phagocytic and engulf neuronal debris as neurons degenerate, a process which is partially regulated by the Draper and Crk/Mbc/dCed-12 signaling pathways. The requirement of one pathway or both is dependent upon the neuronal cell type being phagocytosed. DOI: 10.1101/gad.229518.113 [PubMed: 24361692]

Freeman MR. Drosophila central nervous system glia. Cold Spring Harb Perspect Biol. 2015; 7 pii:a020552. doi: 10.1101/cshperspect.a020552 

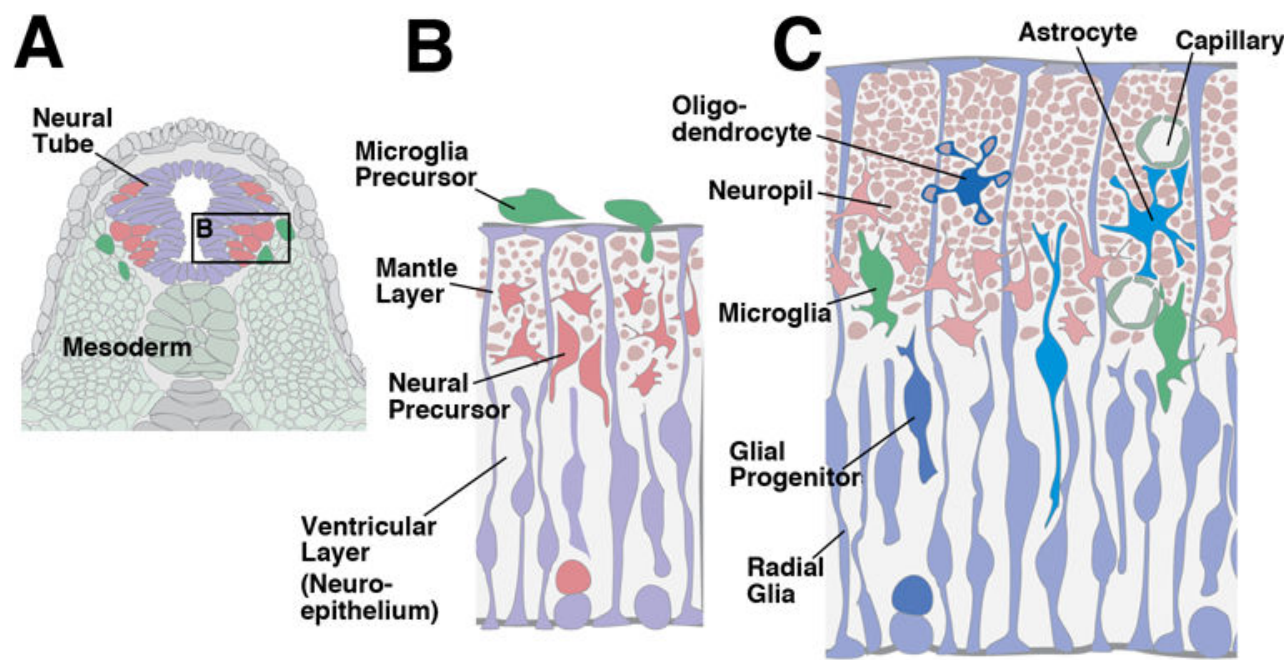

G
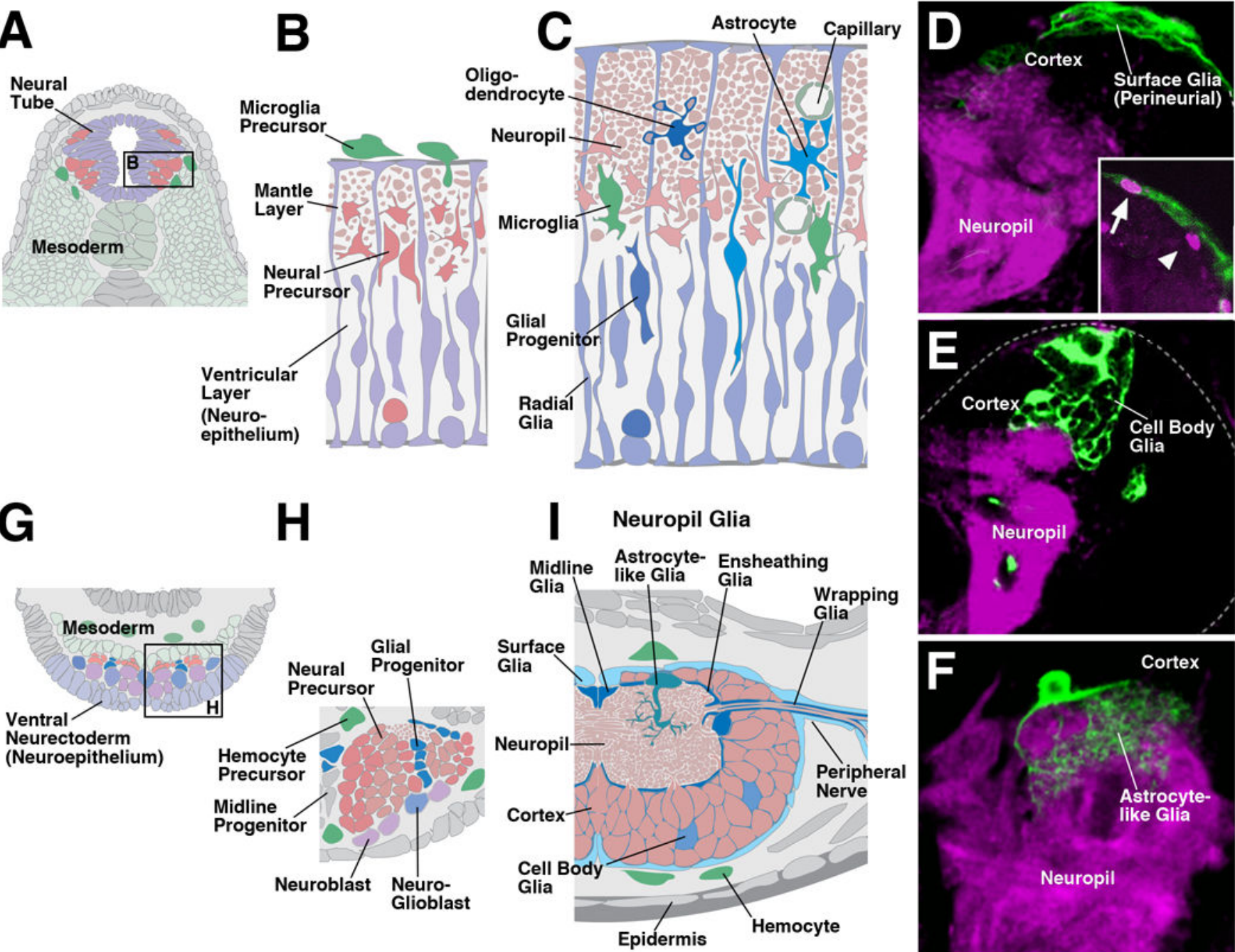

Figure 1.

(A-C) Schematic cross section of vertebrate neural tube at mid-embryo stage (A, B), and around birth (C). Neuroepithelial cells undergo symmetric and asymmetric divisions, thereby generating neural precursors and maintaining their own number. At later stages $(C)$ neuroepithelial cells, now called radial glia, turn to the production of oligodendrocyte progenitors. Other radial glial cells become astrocytes (blue). Microglial cells derived from yolk sac-derived hematopoietic cells spread throughout the mesoderm of the early embryo (A) and infiltrate the neural tube at later stages (B, C). Design of panels (B, C) after Kriegstein and Alvarez-Buylla (2009). (D-F) Types of neuroglia in Drosophila. Frontal confocal sections of late larval brain hemisphere, depicting GFP-labeled MARCM clones of perineurial surface glia (D; 4-cell clone), cortex glia (single cell clone; E), and astrocyte-like neuropil glia (single cell clone; F). Arrow in inset of (D) marks Repo-positive nucleus located within the GFP-labeled perineurial clone; arrowhead points at Repo-positive, GFPnegative nuclei underneath which represent subperineurial glia. In all three panels, the neuropil is labeled with antibody against Drosophila N-cadherin (magenta). (G-I) Schematic cross sections of Drosophila ventral nerve cord at embryonic stages $11(\mathrm{G}), 13(\mathrm{H})$, and 16 (I). Neuroblasts and Neuro-Glioblasts delaminate from the neuroepithelium $(\mathrm{G})$ and give rise 
to fixed neural or neuro-glial lineages by asymmetric division $(\mathrm{H})$. Glial cells then spread out throughout the growing ventral nerve cord and adopt the shape and function of neuropil glia, cortex glia, and surface glia (I). Mesodermally-derived hemocytes migrate along the outer surface of the central nervous system but do not penetrate inside.

Astrocytes ensheath terminal nerve processes and synapses, as well as the capillary network; they form the blood-brain barrier. Oligodendrocytes have large, lamellar processes that assemble into the myelin sheath around long axons in the white matter of the central nervous system (CNS). Similar cells, called Schwann cells, enwrap axons in the peripheral nerves. 


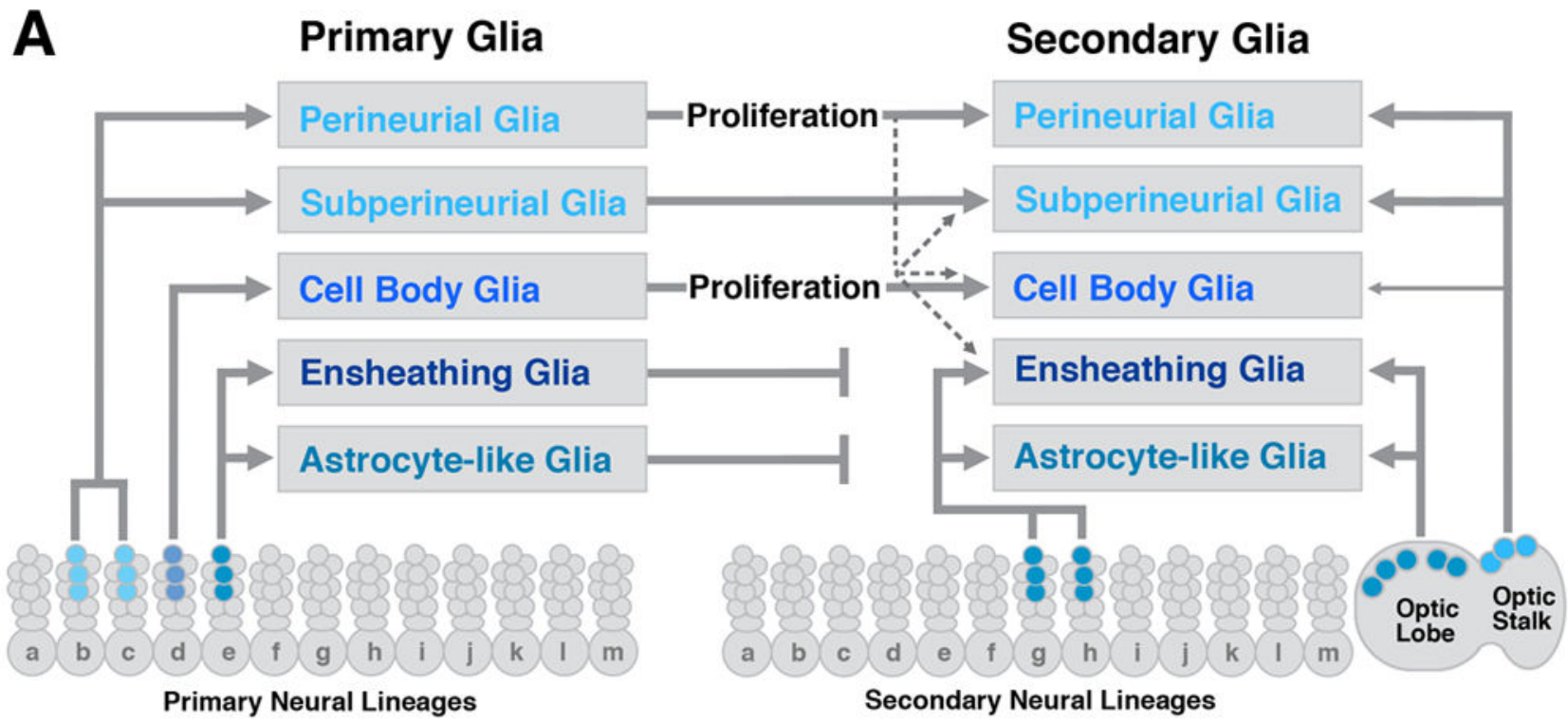

B
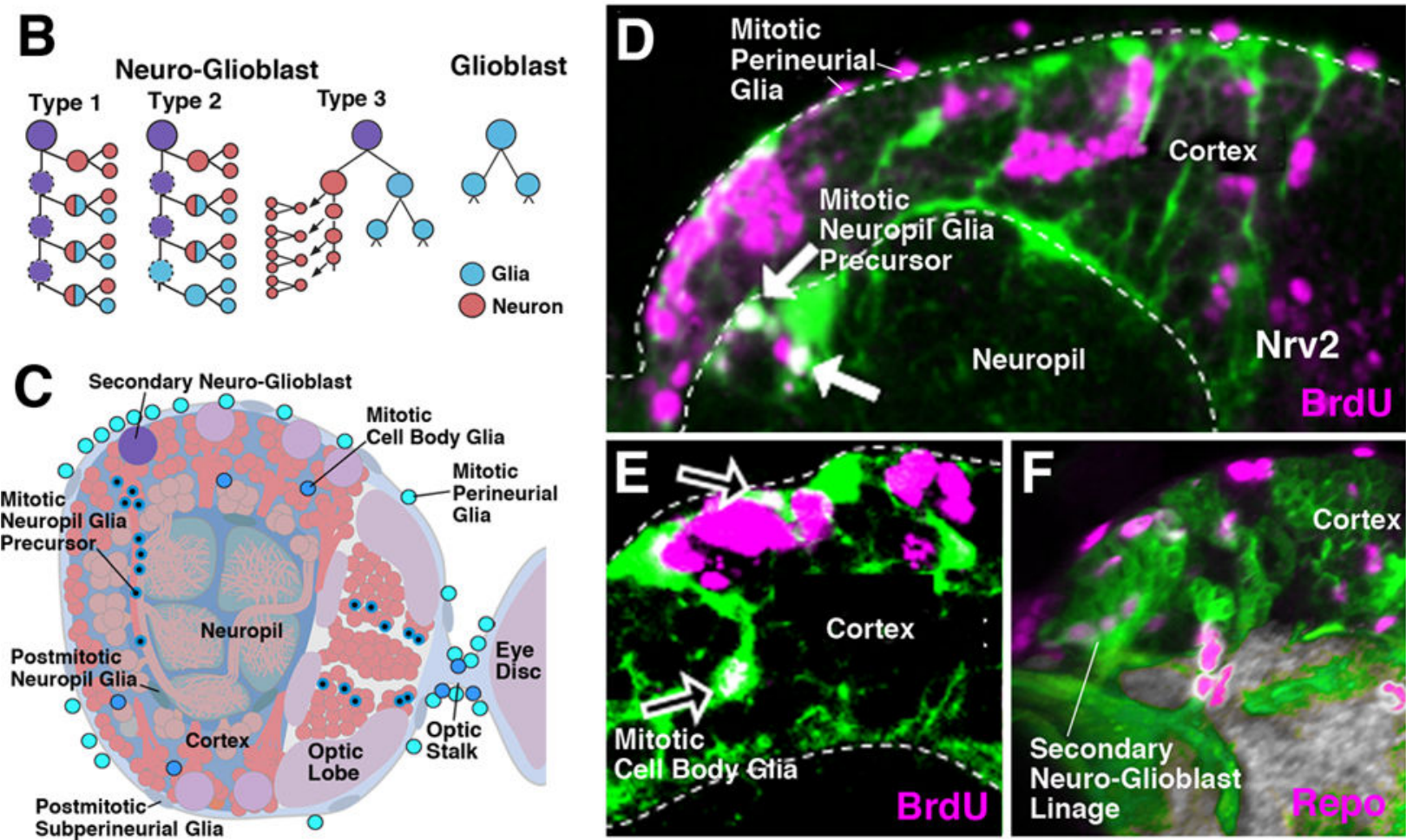

Figure 2.

Origin of primary (larval) and secondary (adult) glia. (A) In the embryo, primary glia arise from a small subset ("b, c, d, e") of proliferating, neural lineages ("a-m"). Primary perineurial glia and cortex glial cells remain mitotically active in the larva and generate secondary glia. Primary neuropil glial cells undergo programmed cell death during metamorphosis and are replaced by glial precursor cells generated from a different set of neural lineages ("g, h") that, along with all other neural lineages, undergo a secondary phase of proliferation during the larval period. Primary subperineurial glial cells most likely are 
retained into the adult period. The primordia of the optic lobe and eye, which proliferate in the larva, contribute a large number of secondary glia of all types. (B) Proliferation pattern of glial progenitors as glioblasts, and type 1-3 neuro-glioblasts (after Udolph et al., 2001; Altenhein et al., 2015). (C) Schematic frontal section of late larval brain hemisphere, showing spatial relationship between differentiated primary glia and secondary glial progenitors. (D, E) Frontal confocal sections of part of late larval brain hemisphere showing proliferating (BrdU-positive; magenta) perineurial glia (D) and cortex glia (E), as well neuropil glia progenitors (D). Nrv-2-Gal4-activated GFP labels cortex glia and neuropil glia (green). (F) Repo-positive neuropil glia progenitors (magenta) form part of secondary lineages, labeled by anti-Neurotactin (green; modified from Omoto et al., 2015). 

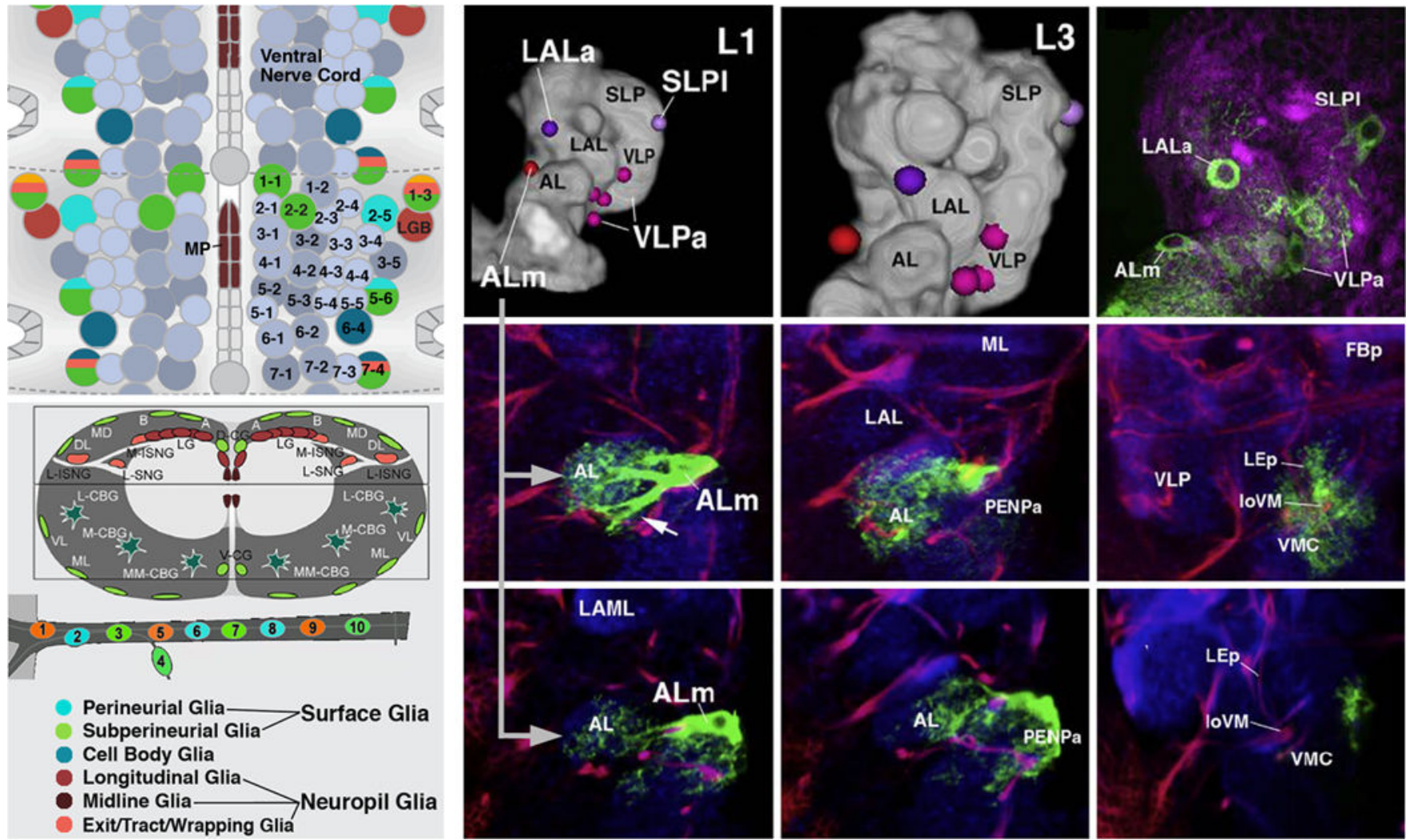

Figure 3.

Fatemap of glial progenitors. (A-C, G) Progenitors of primary glia of the larval ventral nerve cord and peripheral nerves (A-C), and brain (G). (A) Map of the ventral nerve cord with glioblasts and neuro-glioblasts identified by different coloring (after Hartenstein, 2011). Neuroblasts and neuro-glioblasts of one hemineuromere are identified alphanumerically. Lateral glioblast (LGB), Midline progenitors (MP). (B-C): schematic horizontal sections (B, B') and cross section (C) of late embryonic ventral nerve cord and peripheral nerve, showing location of glial cells, identified by same colors as their corresponding progenitors in (A), relative to the boundaries of neuromere, neuropil, and peripheral nerves (modified from Beckervordersandforth et al., 2008; von Hilchen et al., 2011, 2013; Hartenstein, 2011). Boxed areas in (C) indicate dorso-ventral levels of corresponding horizontal sections shown in (B, B'). Bottom of (C): Color key for types of glia used in schematic drawings of panels (A-C, G, K). (D-F) Fate decision between astrocyte-like glia (ALG, green) and ensheathing glia (EG, magenta) depends on Notch activity. Low levels of Notch (E) change structural phenotype of ALG into that of EG (from Peco et al., 2016, with permission). (G) Schematic neuroblast map of the brain, indicating boundaries between the brain neuromeres protocerebrum, deuterocerebrum, and tritocerebrum, and approximate location of glial progenitor clusters giving rise to surface and cortex glia in the protocerebrum (DPSG, VPSG) and deuterocerebrum (ADSG, PDSG), and to neuropil glia (BPLG; neuroblast Td7) (after Omoto et al., 2015). (H-J) Frontal confocal section of brain hemisphere of late larva $(\mathrm{H})$, mid-stage pupa (I), and pharate adult (J), showing development of astrocyte-like glia. Primary astrocyte-like glia (pALG) are lineage-traced from larva onward (white nuclei); these cells are no longer detectable in adult. Secondary astrocyte-like glia (sALG), produced 
in the larva, spread out over the neuropil surface (I) and differentiate in the late pupa (J; reticular processes of pALG and sALG indicated by cyan arrowheads). (K) Fatemap of glia in the optic lobe. Schematic cross sections of larval optic lobe and eye disc (left) and adult optic lobe (right). Nomenclature of optic lobe glia and relationship between larval progenitors and adult glial types is indicated by coloring and arrows. For color code see (C). Abbreviations: CG carpet glia; DSG distal satellite glia; EG epithelial glia; MG marginal glia; NPG neuropil glia; PG perineurial glia; PSUG pseudocartridge glia; SG surface glia; SPG subperineurial glia; XG outer optic chiasm glia 

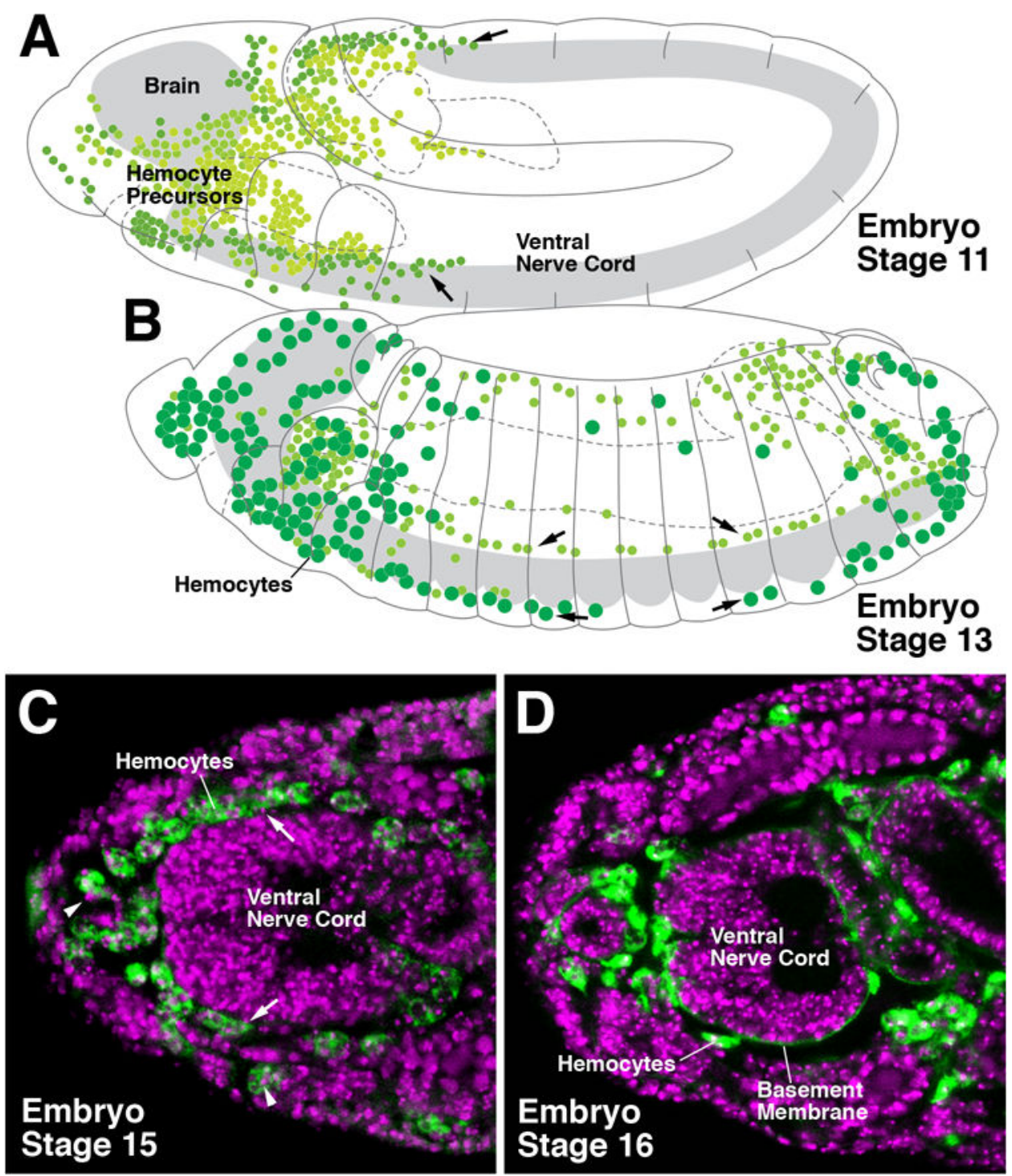

Figure 4.

Origin and migration of hemocytes. (A, B) Drawings of embryos (lateral view) at stage 11 (A) and 13 (B). Profiles of interior structures are shaded (brain, ventral nerve cord) or given as dashed lines (gut primordia). The distribution of hemocyte precursors (small light green circles) and differentiated, phagocytotic hemocytes (large, dark green circles) is indicated, based on camera lucida drawings of anti-Peroxidasin labeled whole mount of representative embryo. Hemocytes migrate along the surface of the CNS (arrows) and other organs (from Tepass et al., 1994, with permission). (C, D) Horizontal confocal sections of anterior ventral nerve cord at embryonic stage 15 (C) and 16 [D; plane of section indicated in (B)]. Nuclei of all cells labeled by Sytox (magenta). Differentiated hemocytes are labeled by antiPeroxidasin; they contain phagolysomes with cellular debris (arrowhead) and are associated with the surface of the ventral nerve cord. By stage 16, hemocytes have deposited a Peroxidasin-positive basement membrane around nerve cord (D). 